\title{
Therapeutic Cycles and Referential Activity in the Analysis of the Therapeutic Process
}

\author{
Rossella Lo Verde $^{1 凶}$, Diego Sarracino ${ }^{1}$, and Marta Vigorelli ${ }^{1}$
}

\begin{abstract}
The present study was designed to show the usefulness of a process analysis based on a joint use of two computerized methods - Mergenthaler's Therapeutic Cycle Model (TCM) and Bucci's Italian Weighted Referential Activity Dictionary (IWRAD). This analysis focused on the transcripts of six sessions from the first eight months of a three-year, face-to-face psychodynamic psychotherapy. Both qualitative and quantitative analyses were conducted. Results showed the presence of specific indicators of a good outcome, according to the two approaches, such as the patient's ability to link reflective processes and felt emotions, the occurrence of referential cycles, and the presence of organized and coherent narratives.
\end{abstract}

Keywords: process analysis, Therapeutic Cycle Model, Referential Activity

The present study proposes an analysis of the therapeutic process based on two computerized instruments for assessing the therapeutic process: Mergenthaler's Therapeutic Cycle Model (TCM; Mergenthaler \& Stinson, 1992), and Bucci's Italian Weighted Referential Activity Dictionary (IWRAD; Maskit, Bucci, Bonfanti, Mariani, \& Visconti di Modrone, 2004). We will introduce the theoretical backgrounds of these instruments, and underline some similarities between them. Then we will examine the role of some key variables that, according to the two approaches, are supposed to be linked with a good psychotherapeutic process. Finally, we will present a single case study, in order to show the evolution of these variables within each session and across several sessions, by focusing on the significant correlations that emerged among TCM and RA variables. This could provide a useful empirical support for examining the similarities and differences between the two theoretical models.

The literature on the TCM (Mergenthaler, 1996, $1998,2000,2003)$ has shown that the presence of a connecting pattern-i.e., the ability to experience appropriate emotional states and, at the same time, to think about them, represents a key aspect of the therapeutic process. According to Karasu (1986),

\footnotetext{
${ }^{1}$ Department of Psychology, University of Milano-Bicocca, Italy.

Correspondence concerning this article should be addressed to Rossella Lo Verde, Department of Psychology, University of Milano-Bicocca, Ed. U6, Piazza dell'Ateneo Nuovo 1, 20126 Milano, Italy. Email: rossella.loverde@virgilio.it
}

when emotional experience and cognitive control are both significantly present in a given time, a change in the patient is observed. Moreover, connecting events were found associated with good treatment outcomes across different therapies and patients (Mergenthaler, 1996; Mergenthaler \& Frost, 2007; Lepper \& Mergenthaler, 2007; Nicolò, Mergenthaler, Pontalti, Semerari, \& Catania, 2000).

Also the presence of several therapeutic cycles plays a central role in the therapeutic process, because in TCM therapeutic cycles represent clinically significant and productive work. Further, Mergenthaler (1998) observed in successful therapies different phases characterized by a decrease of the pattern relaxing -i.e., both low emotional tone and low level of abstraction, which correspond to nonspecific moments that are not directly relevant to the therapeutic process-, and by an increase in both connecting events and number of therapeutic cycles.

The onset of specific fluctuations (i.e., "shift events;" Lepper \& Mergenthaler, 2008; Mergenthaler, 2003) in the patient's emotional state plays an important role during the therapeutic process. These shift events indicate, at a microanalytic level, a change in the quality of the patient's affective experience, which shifts from a deepen-and-provide state, in which the emotional state is predominantly negative and focused on the problem, to a more positive broaden-and-build state, which is focused on the patient's insight and discovery of new opportunities. This transition from a phase of negative experiencing to a more positive one is a necessary precondi- 
tion for connecting. Therefore, we may suppose that the shift events are closely related to the therapeutic change. Mergenthaler (1998) has underlined the active role of the therapist in this process, showing how the therapist can influence the course of treatment by stimulating a series of processes in the patient.

The second method used in this study is the IWRAD (Maskit et al., 2004), a computerized measure of the individual's ability to communicate and transmit his/her own emotional experience to another person. The literature has shown that a good therapeutic process is characterized by an oscillation between a "good hour," related to a high Referential Activity (RA), and a "destabilizing process," involving a low RA (Freedman, Lasky, \& Hurvich, 2002). This may depend on the presencethroughout the treatment, but also within any single session (Bucci, 2005a, b) — of a sequence consisting of an activation phase (characterized by a low RA), a symbolization phase (characterized by a high RA), and a reflective phase (in which the RA decreases again). Recent studies (Fogliato, Strappa, Branchini, \& Rapisarda, 2009) support the hypothesis that the activation of the referential cycle, or even the presence of several "micro-cycles" within any single session, can be considered useful indicators of a wellorganized and well-integrated alternation of the different phases of the referential process.

According to this model, the therapeutic process should encourage a narration of the experience and a reflection (IREF) on it. The referential process can be seen as an alternation of emotional arousal (i.e. the "symbolization" phase, with high IWRAD levels), and narrative restructuring (i.e., the "reorganization" and "reflection" phase, with high IREF levelsi.e., reflexive dictionary). This alternation between symbolization and reflection, which is measured by the IREF/IWRAD negative covariation index, would encourage an improvement of the therapeutic process, by stimulating a better integration between narration and reflection in the patient. In other words, a negative covariation between IREF and IWRAD indicates that the speaker is able to both value words and reflect on his/her experience. In successful therapies, according to Maskit and Bucci (2009), this negative covariation should grow over time.

Some researchers (Bucci \& Maskit, 2008; Mariani \& De Coro, 2009) showed that the RA is usually higher for patients than therapists, since patients' narratives are characterized by higher levels of emotional activation. However, a study by Rivolta (2009) disconfirmed this finding, and concluded that the therapist, in his mirroring process, uses his/her own affective language in order to facilitate the construction of more integrated narrative of the conflictual topics. In contrast, the literature is unanimous in highlighting that the reflection process is higher for therapists than for patients, since therapist have the task of stimulating reflection processes in patients (Bucci \& Maskit, 2008; Mariani \& De Coro, 2009).

\section{Aims of the Study}

The present study aimed to show the utility of a multi-instrumental analysis of the therapeutic process based on the joint use of Mergenthaler's TCM and Bucci's RA. A first purpose of this analysis was to show the similarities between these two approaches. In particular, both instruments (a) use computerized tools and are applicable to written texts; (b) refer to the narrative form rather than on the text content; (c) consider narratives and linguistic styles as important vehicles of the individual's emotional experience, state of mind, and procedural patterns. Therefore, in both approaches it is important that these narratives involve well-integrated and well-connected elements.

As shown previously, several studies suggest that both TCM and RA are important features of the psychotherapeutic process and may indicate a good treatment outcome. In the present study, we examined in detail some key variables that, according to the two approaches, would be at the basis of a good psychotherapeutic process. We used a single-case approach in order to examine the evolution of these variables within each session and across several sessions, and identify the presence of some indicators of a good psychotherapeutic process.

In particular, our study aimed to:

a) Examine the TCM variables during the therapeutic process, by measuring the presence and increase of the connecting pattern and the therapeutic cycles over time, the transition from a negative experiencing (deepen-and-provide) state to a more positive (broaden-and-build) state, and the occurrence of shift events;

b) Assess the trend of IWRAD variables during the therapeutic process. In particular, we aimed to explore the presence of referential micro-cycles (activation, symbolization and reflection) within each session, and the progress of narrative style and IREF/IWRAD covariation index across sessions;

c) Measure the correlations between the TCM and IWRAD dictionaries, in order to assess the similarities and differences between the two approaches.

\section{Method}

\section{Procedure}

The material used in this study is based on the first eight months of a three-year, weekly psychodynamic psychotherapy. Patient's diagnosis was conducted by the psychotherapist herself in the first session, by applying the SWAP-200 (Westen, Shedler, \& Lingiardi, 2003) and the QFM-27 (Albasi, Lasorsa, \& Porcellini, 2007) and administering the SCL-90-R (Derogatis, 1994) to the patient. As regards the process analysis, three two-session blocks were examined, drawn from different phases of the treatment-more specifically, sessions 4-5, 19-20, and 33-34. 
The patient signed informed consent allowing the use of the therapy recordings for clinical and research purposes. The sessions were collected only at the beginning of treatment because, at a certain point of the treatment, the patient asked the therapist to suspend the recording of the sessions because the conversations were more and more intimate and the therapeutic relation was undergoing a turbulent period. Therefore the therapist decided to keep the recordings out of the treatment, in order to guarantee a more collaborative climate.

The audio-recordings of the sessions were transcribed and analyzed according to the TCM and IWRAD standards. In particular, in the case of TCM, the transcriptions were made following standardized transcription rules (Mergenthaler, 1999; Mergenthaler \& Stinson, 1992); then we proceeded to submit them to the Cycle Analysis Model software (Mergenthaler, 1998). As regards Bucci's IWRAD, we applied specific transcription rules for the Italian language (Discourse Attributes Analysis Program; DAAP). Then, we obtained IWRAD (Maskit et al., 2004) and IREF (Mariani \& De Coro, 2009) indices, and a graphical representation of these variables by means of the Systat Software.

\section{Case Presentation}

Bianca is a 29 year-old woman, who requested treatment for anxiety and mild depersonalization states that occurred following the ending of a very problematic romantic relationship. The relationship with her mother was very disorganized and was characterized by aggression, criticism and control. Although the relationship with her father was relatively more stable and positive, overall, Bianca experienced her family as unsupportive and distant. Her experience with her family showed a history of insecure attachment, which may have a role in her internalizing and externalizing problems (e.g., Sarracino, Presaghi, Degni, \& Innamorati, 2011).

At the beginning of the treatment, Bianca depended financially on her parents; however, she showed good coping skills and excellent knowledge resources. She graduated with honors in psychology, enrolled in a master's degree and committed to achieving important academic accomplishments. However, despite these cognitive resources, her ability to manage intimate and romantic relationships was highly inadequate. It was in this domain that Bianca showed many problems.

\section{Instruments}

Shedler-Westen Assessment Procedure-200. (SWAP-200; Westen, Shedler, \& Lingiardi, 2003). This instrument is based on a Q-sort methodology, and is used in the diagnosis and treatment of personality and personality disorders. The 200 items of the SWAP derived from the DSM-IV axis II, and range on a scale from 0 to 7 (from "not descriptive at all" to "very descriptive"); the distribution of items is not free. The SWAP software generates three score profiles. The first profile provides scores for DSM-IV personality disorders (PD Scores). The second provides scores for an alternative set of personality syndromes that were derived empirically through SWAP research (Q-factors). The third provides scores for 12 trait dimensions (factor scores). Several studies (Westen, Shedler, \& Lingiardi, 2003) support the reliability and the predictive validity of the SWAP-200 compared to a series of significant indices, such as personality disorders, hospitalization frequency, suicide attempts, and other clinical measures.

Questionnaire on Mental Functioning. (QFM27; Albasi, Lasorsa, \& Porcellini, 2007). This clinician-report questionnaire consists of 27 items, and is designed to guide the assessment of patients according to the PDM (Psychodynamic Diagnostic Manual) M-Axis (capacity for regulation, attention and learning; capacity for intimacy and relationships; quality of internal experience; capacity for affective experience, expression and communication; defensive patterns and capacities; capacity to form internal representations; capacity for differentiation and integration; self-observing capacities; capacity to construct or use internal standards and ideals) and the three levels of personality organization of $\mathrm{P}$ Axis (Healthy Personalities, NeuroticLevel Personality Disorders and Borderline-Level Personality Disorders).

Symptom Checklist-90-Revised. (SCL-90-R; Derogatis, 1994). This self-report scale is composed of 90 items that represent the more common symptoms observed in psychiatric patients. It assesses 9 primary symptomatic dimensions: somatization, obsessivity, compulsiveness, sensitivity to interpersonal relationships, depression, general anxiety, hostility, paranoid ideation, and psychoticism. The scale also provides 3 additional psychopathological indices: General Severity Index (GSI), Positive Symptoms Distress Index (PSDI), and Positive Symptoms Total (PST).

The Therapeutic Cycles Model. (TCM, Mergenthaler, 1996, 1998, 2000, 2003). This instrument is grounded on the Resonating Mind Theory (RMT), a clinical theory about the therapeutic change, which is based on a computerized method that allows the detection of clinically significant aspects in each session or during a whole treatment course. It refers to two different change variables (Karasu, 1986), i.e., "emotion" and "abstraction". These two factors are combined together and give rise to four emotion-abstraction patterns; the most important one, in the perspective of change, is "connecting", i.e., the ability to get into personal emotional states and, in the meantime, think about them. In optimal 
cases, these patterns follow a specific chronological sequence, called therapeutic cycle, which consists of different phases: relaxing (characterized by low emotional tone and low abstraction), experiencing (when patient's emotions are aroused), connecting (when both emotional tone and abstraction are high), reflecting (when the patient starts to think about its feeling), and then relaxing again. Mergenthaler (1996) suggests that a therapeutic cycle is a clinically significant event.

Therapeutic cycles are highlighted by a method consisting of two parts: a previous transcription based on standard criteria (Mergenthaler, 1999; Mergenthaler \& Stinson, 1992) and a proper computerized analysis that uses a specific software (Mergenthaler, 1998). This software can calculate emotion-abstraction patterns and therapeutic cycles thanks to two dictionaries that measure the frequency of emotion and abstraction words. The emotional tone dictionary includes all those words (adjectives, adverbs or verbs) having a positive or negative value. The abstraction dictionary, instead, includes words indicating general categories of objects or entities. In addition, another dictionary measures the narrative style, i.e., different narration modalities to tell past events throughout specific time connections.

Some empirical studies support the principles of RMT and data issued from TCM. In particular, as said above, some results underline how connecting can be considered as the go-between of the therapeutic change, since significant correlations emerged between the psychotherapeutic intervention and the connecting, and between the connecting and the therapeutic change (Mergenthaler \& Gelo, 2007; Nicolò et al., 2000).

Referential Activity Linguistic Measures. (RA, Maskit et al., 2004). This method is based on Wilma Bucci's Multiple Code Theory (Bucci, 1997). This theory of mental functioning identifies three ways to elaborate information: symbolic verbal code, symbolic non-verbal code, and non-symbolic code. These formats are included in the so-called Referential Process, which is operationalized in terms of Referential Activity (RA) - i.e., the ability to express one's non-verbal, emotional or somatovisceral experience in words. In particular, according to Bucci, in a therapeutic process we can observe a referential cycle consisting of an arousal phase, characterized by a low RA, a symbolization phase, related to a high RA, and a reflection phase, where RA becomes lower (Bucci, 1999). Nevertheless, recent studies have revealed the presence of micro-cycles in any single session, rather than one homogeneous referential cycle (Fogliato et al., 2009).

RA is assessed using a manualized codifying system (Bucci \& Mc Kay, 1992), and since 2004 has been applied together with a computerized method, the IWRAD dictionary (Maskit et al., 2004). This method analyzes session narratives and compares every word with the occurrences listed in its dictionary. The IWRAD produces micro- and macro-analyses for each session, and calculates a series of indices referring to specific dictionaries-e.g., not-fluent (DF), ref lexive (IREF), and affective (IAFF) words.

\section{Results}

\section{DSM-IV, SWAP-200, QFM-27, and SCL-90-R Diagnoses}

At the beginning of the treatment, Bianca's DSMIV-TR (APA, 2000) diagnosis was Anxiety Disorder Not Otherwise Specified (Axis I). Regarding Axis II, in the SWAP-200 Bianca obtained a Q-score of 60.56
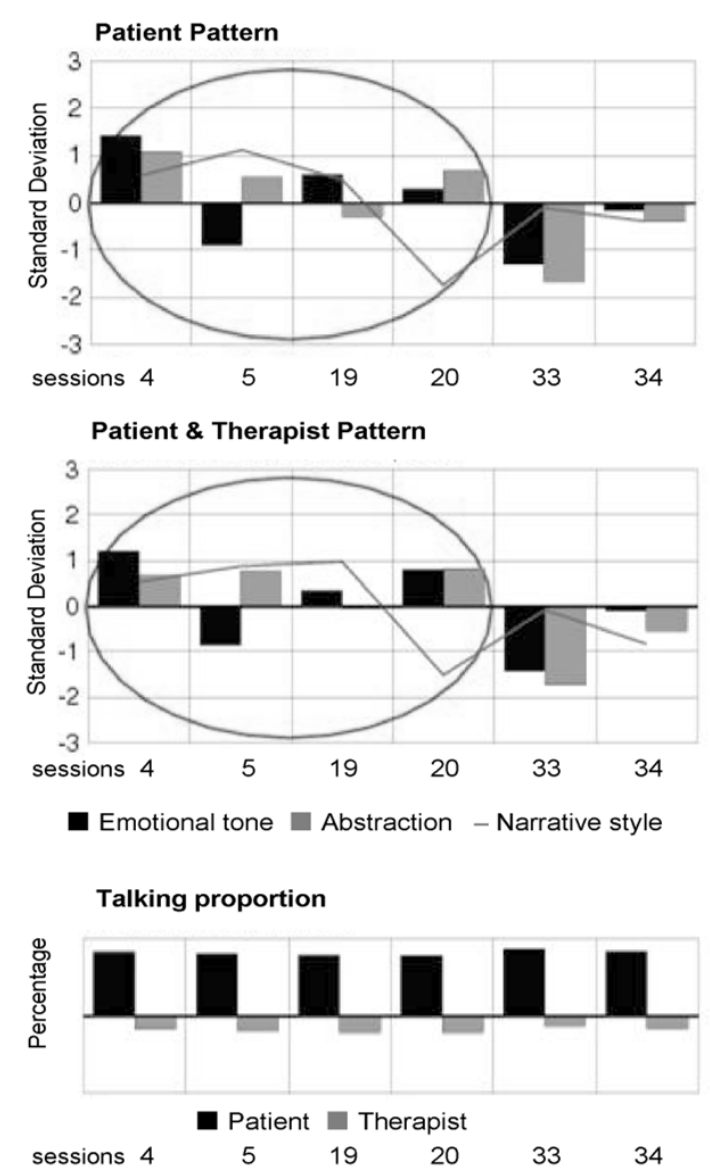

Emotional tone (Patient \& Therapist)

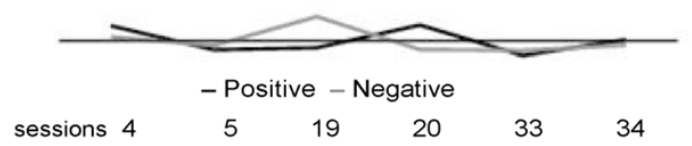

Figure 1. Analysis of six sample sessions in the first eight months of Bianca's treatment. The first graph shows the trend of patient emotion-abstraction patterns. The second graph shows the trend of patient/therapist emotion/abstraction patterns. In both graphs, the $\mathrm{x}$-axis represents the sessions and the y-axis represents the standard deviations from the mean. The circle indicates a therapeutic cycle. The third graph shows, in percentage, the proportion of words spoken by patient and therapist. The fourth graph represents the trend of positive and negative emotional tones for both therapist and patient. 
Table 1. Comparison between different groups of sessions in relation to connecting patterns, therapeutic cycles and RA indexes

\begin{tabular}{|c|c|c|c|c|c|c|c|}
\hline & \multicolumn{7}{|c|}{ Session block } \\
\hline & A & B & $\mathrm{C}$ & $\mathrm{A} v s \mathrm{~B}$ & $\mathrm{~A} v s \mathrm{C}$ & $\mathrm{B}$ vs $\mathrm{C}$ & $\mathrm{B} v s \mathrm{AC}$ \\
\hline & Mean $(S D)$ & Mean $(S D)$ & Mean $(S D)$ & $t$ & $t$ & $t$ & $t$ \\
\hline Connecting $\mathrm{P} \& \mathrm{~T}$ & $.26(.44)^{\mathrm{a}}$ & $.32(.47)^{\mathrm{b}}$ & $.27(.44)^{c}$ & -1.004 & -0.151 & 0.855 & -1.080 \\
\hline Connecting $\mathrm{P}$ & $.20(.40)^{\mathrm{a}}$ & $.21(.41)^{\mathrm{b}}$ & $.27(.45)^{c}$ & -0.204 & -1.265 & -1.042 & 0.499 \\
\hline Therapeutic Cycles P \& T & $.38(.49)^{\mathrm{a}}$ & $.45(.50)^{\mathrm{b}}$ & $.35(.48)^{c}$ & -1.029 & 0.553 & 1.578 & -1.507 \\
\hline Therapeutic Cycles P & $.30(.46)^{\mathrm{a}}$ & $.24(.43)^{\mathrm{b}}$ & $.37(.48)^{c}$ & 1.123 & -0.989 & $-2.103^{*}$ & 1.831 \\
\hline IWRAD P & $.49(.05)^{\mathrm{d}}$ & $.49(.03)^{\mathrm{e}}$ & $.50(.03)^{\mathrm{f}}$ & 0.221 & 0.011 & $-2.464^{*}$ & 1.078 \\
\hline IREF P & $.02(.07)^{\mathrm{d}}$ & $.02(.04)^{\mathrm{e}}$ & $.02(.05)^{\mathrm{f}}$ & $2.458^{*}$ & $2.300^{*}$ & -0.192 & 1.735 \\
\hline IREF/IWRAD P & $-.01(.38)^{\mathrm{g}}$ & $-.04(.40)^{\mathrm{h}}$ & $.00(.38)^{\mathrm{i}}$ & $2.489^{*}$ & -0.819 & $-3.070^{*}$ & $3.118^{*}$ \\
\hline
\end{tabular}

Note. Block A = sessions 4-5; Block $\mathrm{B}=$ sessions 19-20; Block $\mathrm{C}=$ sessions 33-34. $\mathrm{P}=$ patient; $\mathrm{T}=$ therapist; IWRAD = Italian Weighted Referential Activity Dictionary; IREF = Italian Reflection ictionary; IREF/IWRAD = IREF/IWRAD negative covariation index.

${ }^{\mathrm{a}} n=112 .{ }^{\mathrm{b}} n=106 .{ }^{\mathrm{c}} n=112 .{ }^{\mathrm{d}} n=931 .{ }^{\mathrm{e}} n=610 .{ }^{\mathrm{f}} n=654 .{ }^{\mathrm{g}} n=1862 .{ }^{\mathrm{h}} n=1219 .{ }^{\mathrm{i}} n=1312$.

$* p<0.05$

for the histrionic personality trait and 60.96 for the high-functioning depressive trait.

According to the QFM-27, the patient showed various problems related to intimate relationships. The percentages related to her mental functioning were $45.76 \%$ neurotic, $27.12 \%$ deficiency, and $27.12 \%$ resources. More specifically, Bianca's most problematic area was her ability to form intimate relationships, due to her feelings of ambivalence, and her difficulty in understanding the consequences of the interactive behavior that might affect her involvement in intimate relations.

Finally, the SCL-90-R revealed high scores ( $\mathrm{T}>50)$ on the "depression", "hostility" and "paranoid ideation" scales.

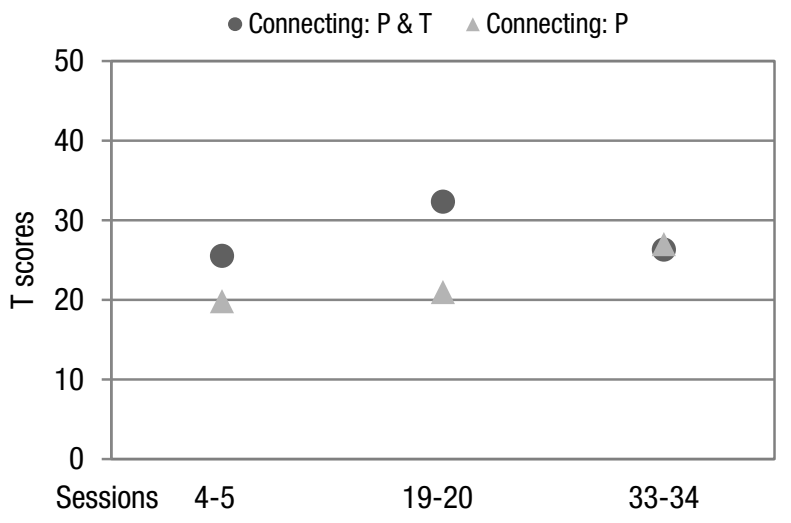

\section{Analysis of the Therapeutic Cycles}

The TCM analysis (Figure 1) shows the evolution of the emotion-abstraction pattern, and allowed us to identify the presence of key moments in Bianca's treatment. As regards the progress of the connecting pattern and the number of treatment cycles in the course of time, we did not observe a steady increase of these variables in the therapeutic dyad, but rather a more complex pattern (Figure 2).

As regards the percentage of the patient/therapist connecting, there is a "peak" in correspondence with the central sessions, in particular session 20. It should be noted that the percentage of patient's connecting is significantly lower (Table 1 ) in sessions 4-5 and

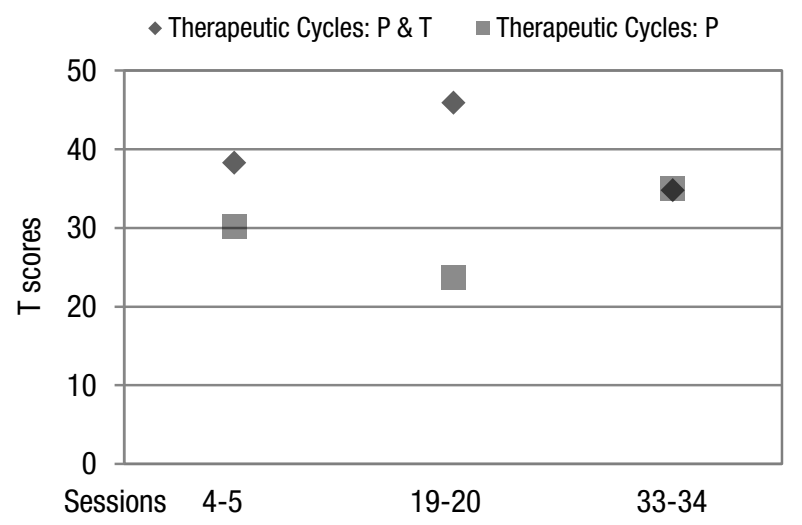

Figure 2. Connecting patterns and therapeutic cycles. The first graph shows, in $\mathrm{T}$ scores, the presence of the connecting pattern in patient/therapist $(\mathrm{P} \& \mathrm{~T}$ ) and patient $(\mathrm{P})$ narratives, during the three two-session blocks considered. The second graph shows the therapeutic cycles in patient/therapist $(\mathrm{P} \& \mathrm{~T})$ and patient $(\mathrm{P})$ narratives. 

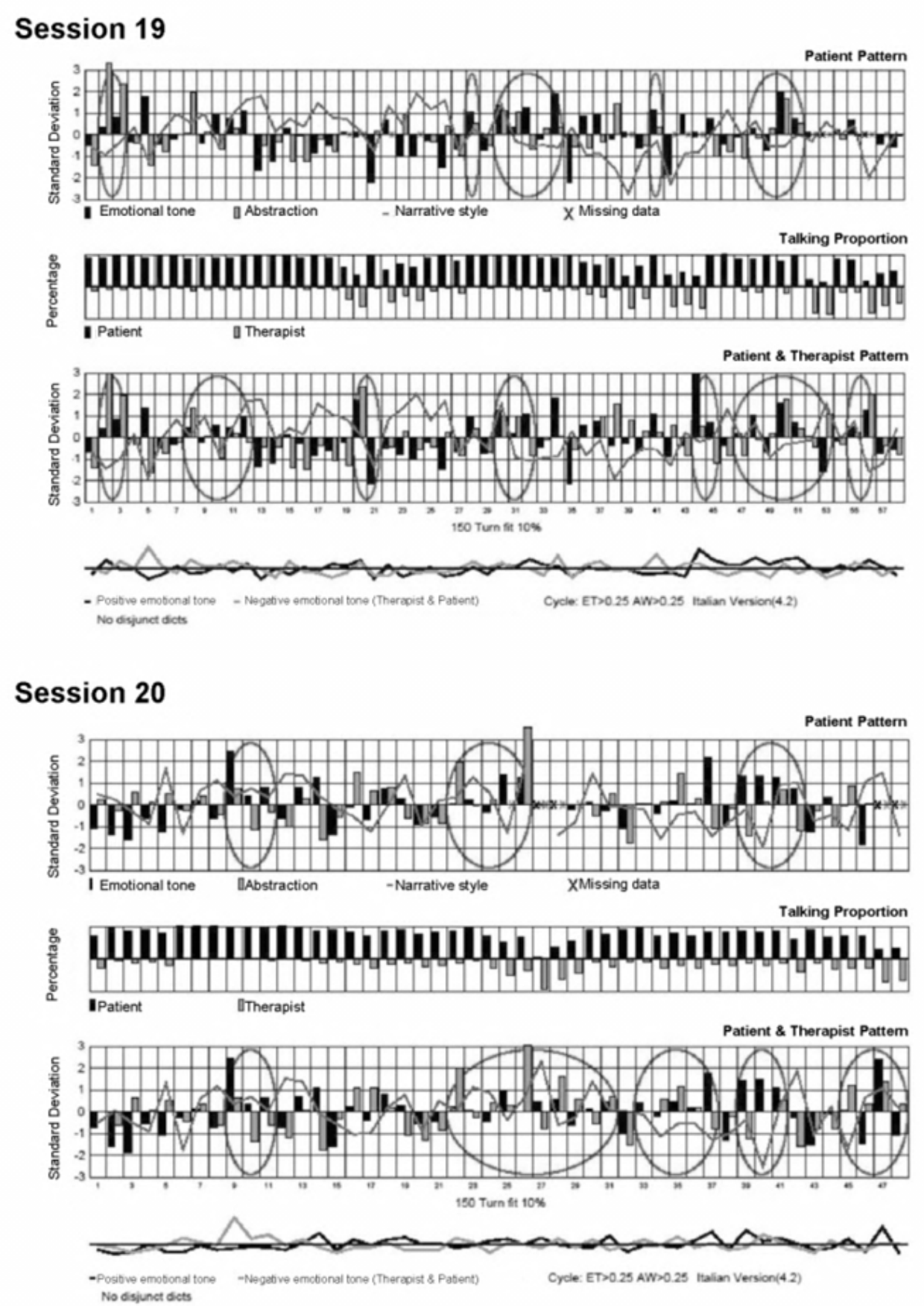

Figure 3. Microanalysis of the therapeutic cycles: comparison between sessions 19 and 20. The figure shows the graphical output of two relevant sessions.

19-20, and "realigns" with the patient-therapist connecting only in sessions 33-34.

We observed a similar trend, even more pronounced, in the percentage of treatment cycles. Also in this case, the patient/therapist score forms a peak in correspondence with sessions 19-20, while the percentage of therapeutic cycles relating to patient's verbalizations is lower (Table 1). However, once again, it "realigns" with the patient-therapist score in sessions 33-34. A more detailed analysis of the sessions examined shows a pervasive presence of connecting events and treatment cycles. Figure 3 shows the graphical output of two relevant sessions.
In session 19 we observe a high narrative style, several shift events and a peak of negative emotions. This may have laid the groundwork for those signs of change detected in session 20 , characterized by very positive emotions. Following is an example of a connecting episode, drawn from session 20:

T: So, this impossibility to relate [to her boyfriend] looks like other situations in which you felt ineffective and powerless.

$\mathrm{P}: \mathrm{Mmh} /$ hesitation (00:00:05) . . very often when I try to talk to my parents.

$\mathrm{T}$ : Aha/exclamation.

P: Generally speaking. 
$\bullet$ IWRAD P

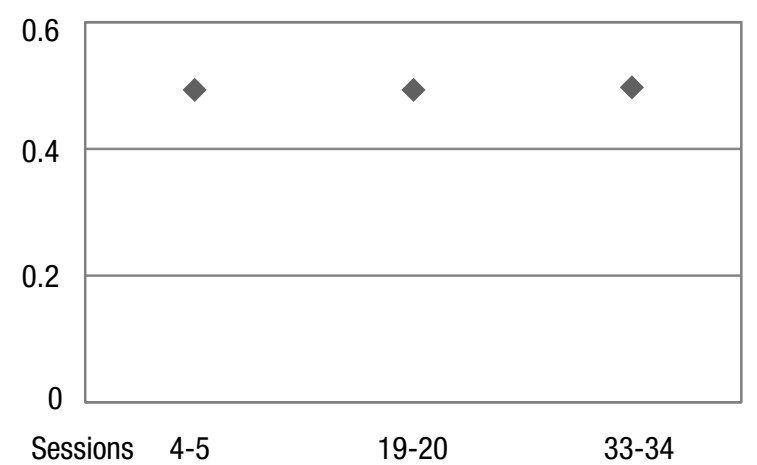

$\square$ IREF P $\triangle$ Covar IWRAD/IREF

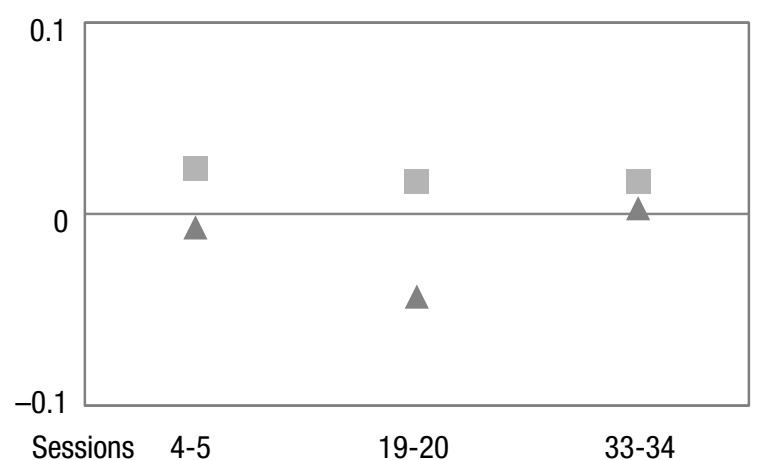

Figure 4. Referential Activity indexes. IWRAD = Italian Weighted Referential Activity Dictionary; IREF = Italian Reflection Dictionary; IREF/IWRAD = IREF/IWRAD negative covariation index. The first graph shows a comparison between the patient's IWRAD values among the three session blocks considered. The second graph shows the IREF values and IREF/IWRAD covariation index in the same session blocks.

In this passage, the therapist asks the patient to reflect on the feeling of powerlessness that she feels when trying to relate to her boyfriend, and allows her to link the relationship with her boyfriend with her family situation. Notably, the patient needs a few seconds to fully understand this.

The presence of connecting events is concentrated mainly in session 20. More in detail, there is a transition between sessions 19 and 20 from a deepen-and-provide state, characterized by negative experiencing, to a broaden-and-build state, characterized by positive emotions (Figure 3). The deepenand-provide state is stimulated by therapeutic interventions such as the following:

T: Let's stop on the first point, that [your boyfriend] is unwell, and the very fact that not feeling good leads him to the end of his resources. How do you think a person may feel in this state?

On the other hand, the broaden-and-build state is encouraged by therapeutic interventions such as the following:

$T$ : And yet there was a need for a series of factors, including the fact that ... you are obtaining academic success ... and you are starting to open up to others.

Also from a clinical point of view, session 19 appears to be a turning point, which seems to activate in Bianca the ability to make decisions and live out her experiences; this appears to announce the change observed in session 20 (Figure 3). Session 20, in fact, introduces Cristina, a person who will become very important in Bianca's life. The patient felt that she had finally found a friend, and her ability to cope with her relational issues improved. This change in her ability to express positive emotions and connect with emotional and cognitive processes may depend not only on this close friendship but also on a new way of seeing her relational issues. In fact, now she starts to put her parents in the back- ground and have more respect for herself and their current relationships.

\section{Analysis of the Referential Activity}

The RA analysis shows that the IWRAD does not increase regularly during the sessions. There is, however, a slight increase in the patient's narrative ability toward the end of this initial treatment period (Figure 4).

If we consider the IREF trend (i.e. those words that suggest "how people think and communicate thoughts"), we can see that it is higher in sessions 4 and 5 compared to subsequent sessions. This presence of reflective processes in the early stages of the treatment may represent a defensive attitude on the part of the patient, who could now use her efficient cognitive functioning to cope with her problems.

Finally, the trend of the IREF/IWRAD covariation

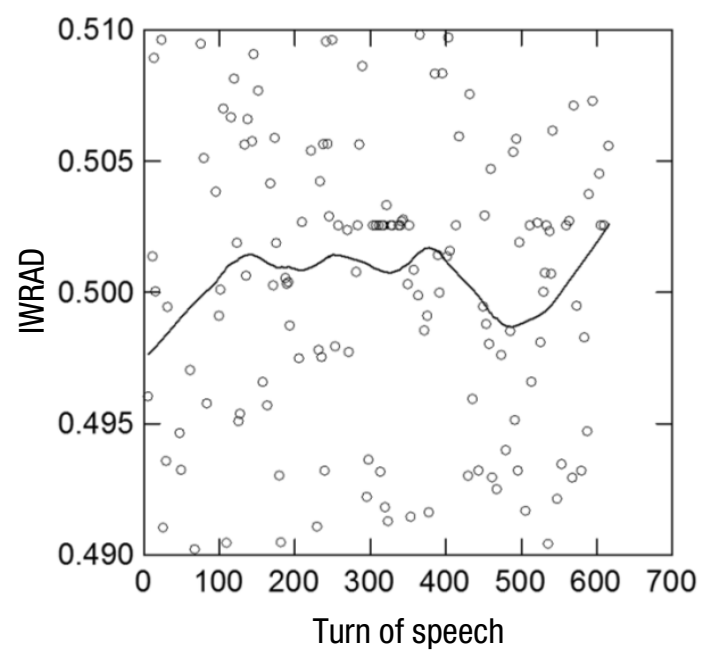

Figure 5. IWRAD trend within session 20. IWRAD = Italian Weighted Referential Activity Dictionary. In this graph, the $x$-axis represents the turn of speech, the $y$ axis represents the IWRAD within session 20 . 
index shows that sessions 19-20 present higher values than sessions 33-34. Therefore, the negative covariation in our sample of sessions does not increase over time.

Within each session, we can see the occurrence of multiple micro-cycles rather than a single referential cycle. Figure 5 shows the trend of these microcycles during session 20 across its speech turns. As we can see from the graph, after a slight rise in the IWRAD value, we can identify the presence of three referential cycles.

\section{Comparison between Therapeutic Cycles and Referential Activity Dictionaries}

Comparing TCM and IWRAD dictionaries that share similar theoretical definitions, we observed a positive correlation between Mergenthaler's abstract words and Bucci's reflective words $(r=.32, p<.001)$, a higher positive correlation between Mergenthaler's emotional dictionaries and Bucci's affective words, especially for the positive words $(r=.60, p<.00)$, and a weak but significant positive correlation between Mergenthaler's narrative style and Bucci's $\operatorname{IWRAD}(r=.14, p<.01)$.

\section{Discussion}

In conclusion, the instruments for assessing therapeutic process used in this study where able to show, session by session, some indices of a good therapeutic process hypothesized by TCM and RA approaches, e.g. the presence of a connecting pattern. In our data, this pattern emerged in several sessions, and this, according to the previous literature (Mergenthaler, 1996, 1998, 2000, 2003), may represent a clear sign of the presence of both intense emotional experience and high cognitive resources in the patient.

In contrast, the connecting did not show a steady increase, but a more complex pattern. In particular, the patient-therapist connecting score showed a "peak" in sessions 19-20, whereas the patient's connecting score was lower in the initial and middle sessions, and "realigned" only in the later sessions examined in our study.

It is important to mention a limitation of this study, i.e. the small number of sessions examined. In fact, a complete session-by-session analysis (e.g. Sarracino \& Dazzi, 2007) should improve the opportunity to detect any change and micro-change in the therapeutic process. Unfortunately, at a certain point of the treatment, the patient asked the therapist to suspend the recording of the sessions because their conversations were more and more intimate and the therapeutic relation was undergoing a turbulent period. The therapist then decided to keep the recordings out of the treatment, because by agreeing to the patient's request she was able to restore a more collaborative climate.

Another limitation of the study is due to the fact that the first eight months of a long-term psychotherapy may be too short a period to notice a significant improvement in the patient with this specific assessment procedure. Moreover, we should consider that the six sessions examined are not consecutive. Sessions 33 and 34, for example, followed a break due to summer vacation: The absence of connecting, reflecting or experiencing patterns, therefore, may depend on the fact that more sessions were needed to restore a fully productive clinical work.

Moreover, it is also possible that the connecting pattern increases physiologically after a specific phase in which this ability rapidly declines. It is unlikely, in fact, that a linear and constant increase of the connecting pattern is essential to the change; in other words, we suppose that this improvement might occur in several ways, and these different modalities might constitute an interesting argument for future research.

In our set of sessions, the transition from a deepenand-provide state - characterized by a negative experiencing pattern - to a broaden-and-build statecharacterized by more positive feelings, emerged in the middle sessions. This may indicate that these sessions may have a clinical relevance in this study. The higher number of shift events of session 19 might have a role in promoting the therapeutic change (Lepper \& Mergenthaler, 2008; Mergenthaler, 2003). Indeed, shift events indicate, on a micro-analytic level, the presence of a change in the quality of the patient's affective experience, which shifts from an emotional state, mostly focused on patient's difficulties, to a state oriented to the insight and the discovery of new opportunities.

By examining the IWRAD trend, we observed the presence of several referential micro-cycles in each session considered. According to previous studies (e.g. Freedman et al., 2002), this may indicate a good functioning of the therapy, because it implies a correct alternation of the different phases of the referential process-activation, symbolization, and reflection. The orderly progression of these phases observed in our set of sessions, particularly in sessions 19-20, shows that it was possible to elicit in the patient emotional patterns and experiences that were dissociated from their symbolic meaning, to activate a link between the sub-symbolic components and the words, and to reflect on the meanings of the stories narrated and shared by the patient.

Moreover, the affective component of the therapeutic relationship helped the patient to improve the complexity of her self-account, as suggested by previous studies (e.g., Mariani \& De Coro, 2009). In fact, in the 8-month period considered in this study, we observed an increase, although slight, of the patient's IWRAD and narrative abilities.

Finally, the negative covariance between IREF e 
IWRAD is higher in the "middle" sessions; in contrast, the later sessions examined in this study showed a lower negative covariance, contrary to previous literature. Once again, this might depend on the fact the our data does not refer to the entire treatment but to the first eight months, and this period might be too short to observe such an improvement.

Our study showed, in a preliminary way, the usefulness of a joint use of TCM and IWRAD, two computerized methods that share many characteristics and, therefore, may be considered mutually enriching and, in part, interchangeable. In fact, both TCM and IWRAD are focused on the linguistic style rather than a content analysis. The language used in the narratives is considered an important vehicle of unconscious thought, states of mind, and emotional experiences.

Both TCM and IWRAD require that these narratives are well integrated and connected, although with some differences. In particular, for TCM this linking concerns the ability to experiment an emotional state and, in the same time, to be able to reflect on it. In contrast, for IWRAD this integration is between the verbal and non-verbal experience.

Both Mergenthaler's therapeutic cycle and Bucci's referential cycle are regarded as key aspects of the therapeutic process and indices of a good outcome. Besides the fact that both the instruments are based on the findings and the concepts derived from cognitive psychology and neuroscience, it is important to note another similarity: also TCM includes a dictionary specifically designed to assess referential activity (Mergenthaler \& Bucci, 1999). In fact, Mergenthaler originally worked with Bucci in a preliminary version of the IWRAD, called the "Computerized measure of the Referential Activity" (CRA; Mergenthaler \& Bucci, 1999). In the CRA, the referential activity was considered as an index of the narrative style, although not equivalent to it. Moreover, the DAAP software calculates some indices of the affective and reflective words that may be compared with TCM's emotion tone and abstraction. In our study, this comparison showed significant positive correlations between Mergenthaler's abstract words and Bucci's affective words, and between Mergenthaler's narrative style and Bucci's IWRAD. The relatively low values, although significant, of these correlations may depend on an imprecise matching between the two dictionaries, which shows how important is to use both the instruments to cover different aspects of the therapeutic process.

\section{References}

Albasi, C., Lasorsa, C., \& Porcellini, E. (2007). QFM-27: Questionario sui livelli di funzionamento mentale [QFM-27: Questionnaire on the levels of mental functioning]. Retrieved from http://www.pdm-qfm.com

American Psychiatric Association (2000). Diagnostic and statistical manual of mental disorders: Text revision (DSM$I V$-TR). Washington, DC: Author.

Bucci, W. (1997). Psychoanalysis and cognitive science. New York: Guilford Press.

Bucci, W. (1999). Schemi del discorso nelle ore "buone" e in quelle problematiche: Un'interpretazione tramite il codice multiplo [Patterns of speech in "good" and problematic hours: An interpretation based on the multiple code]. Psicoterapia, 14/15, 30-47.

Bucci, W. (2005a). Process research. In E. Person, A. Cooper \& G. Gabbard (Eds.), Textbook of psychoanalysis (pp. 317-333). Washington, DC: American Psychiatric Press.

Bucci, W. (2005b). Basic concepts and methods of psychoanalytic process research. In E. Person, A. Cooper \& G. Gabbard (Eds.), Textbook of psychoanalysis (pp. 339355). Washington, DC: American Psychiatric Press.

Bucci, W., \& Maskit, B. (2008, January). Computerized views of psychoanalytic treatments at increasing magnification. RAAPA Meeting, New York.

Bucci, W., \& Kabasakalian-Mckay, R. (1992). Scoring referential activity: Instructions for use with transcripts of spoken narrative texts. Ulm: Ulmer Textbank.

Derogatis, L. R., (1994). SCL-90-R: Administration, scoring and procedures manual. Minneapolis: National Computer Systems.

Fogliato, E., Strappa, V., Branchini, S., \& Rapisarda, F. (2009). L'attività referenziale [The referential activity]. In M. Vigorelli (Ed.), Laboratorio didattico per la ricerca in psicoterapia. [Training course in psychotherapy research] (pp. 275-310). Milano: Raffaello Cortina.

Freedman, N., Lasky, R., \& Hurvich, M. (2002, September). Two pathways toward knowing psychoanalytic process. $\mathrm{Pa}-$ per presented at the IPA conference "Pluralism of sciences: The psychoanalytic method between clinical, conceptual and empirical research", Frankfurt, Germany.

Karasu, T. B. (1986). The specificity versus nonspecificity dilemma: Toward identifying therapeutic change agents. American Journal of Psychiatry, 143, 687-695.

Lepper, G., \& Mergenthaler, E. (2007). Therapeutic collaboration: How does it work? Psychotherapy Research, 17, 576-587.

Lepper, G., \& Mergenthaler, E. (2008). Observing therapeutic interaction in the "Lisa Case". Psychotherapy Research, 18(6), 634-644.

Mariani, R., \& De Coro, A. (2009). Il dizionario delle parole riflessive (IREF): una misura linguistica nello studio del processo referenziale su tre casi singoli [The dictionary of reflective words (IREF): A linguistic measure in the study of referential process of three single case]. Quaderni CPD, 6, 25-33.

Maskit, B., \& Bucci, W. (2009). Confronto tra misure computerizzate del processo referenziale in psicoterapie psicoanalitiche [Comparison between computerized measures of referential process in the psychoanalytic psychotherapy]. Quaderni CPD, 6, 17-24.

Maskit, B., Bucci, W., Bonfanti, A. A., Mariani, R., \& Visconti di Modrone, E. (2004, June). Making and using the Italian Weighted Referential Activity Dictionary (IWRAD). Paper presented at the $35^{\text {th }}$ International Meeting of the Society for Psychotherapy Research, Roma, Italy.

Mergenthaler, E. (1996). Emotion-abstraction patterns in verbatim protocols: A new way of describing psychotherapy process. Journal of Consulting and Clinical Psychology, 64, 1306-1315.

Mergenthaler, E. (1998). I patterns di emozione-astrazione nei trascritti delle verbalizzazioni: un nuovo approccio per la descrizione dei processi in psicoterapia [The emotion-abstraction patterns in the transcribed verbalizations: A new approach for describing psychotherapy pro- 
cesses]. Psicoterapia, 12, 26-38.

Mergenthaler, E. (1999). Regole standardizzate di trascrizione delle sedute di psicoterapia [Standardized rules of transcription of psychotherapy sessions]. Psicoterapia, 14/15, 21-29.

Mergenthaler, E. (2000). The therapeutic cycle model in psychotherapy research: Theory, measurement, and clinical application. Ricerche sui Gruppi, 5(10), 34-40.

Mergenthaler, E. (2003, June). Shifts from negative to positive emotional tone: Facilitators of therapeutic change? Paper presented at the $34^{\text {th }}$ International Meeting of the Society for Psychotherapy Research, Weimar, Germany.

Mergenthaler, E., \& Bucci, W. (1999). Linking verbal and non-verbal representations: computer analysis of referential activity. British Journal Medical Psychology, 72(3), 339-354.

Mergenthaler, E., \& Gelo, O. (2007). Un'analisi qualitativa del disturbo di personalità narcisista attraverso il modello del ciclo terapeutico: uno studio single-case (Caso K.) [A qualitative analysis of narcissistic personality disorder using the therapeutic cycle model: A single-case study ( $\mathrm{K}$. case)]. In G. Nicolò \& S. Salvatore (Eds.), La Ricerca in Psicoterapia [Research in Psychotherapy] (pp. 329-353). Roma: Edizioni Carlo Amore.

Mergenthaler, E., \& Stinson, C. H. (1992). Psychotherapy transcription standards. Psychotherapy Research, 2, 125-142.

Nicolò, G., Mergenthaler, E., Pontalti, I., Semerari, A., \&
Catania, D. (2000). Analisi dei Patterns di emozioneastrazione in un trattamento cognitivista: Risultati preliminari [The analysis of Emotion-Abstraction Patterns in a cognitive treatment: Preliminary results]. Psicoterapia, $21,67-72$.

Rivolta, L. (2009). Il processo referenziale nei colloqui del processo diagnostico [The referential process in the interviews during the diagnostic process]. Quaderni CPD, 6 , 85-99.

Sarracino D., \& Dazzi N. (2007). Motivational Areas Rating Scale: Developing a new tool for studying psychotherapy process and outcome. Psychotherapy Research, 17(6), 721732.

Sarracino, D., Presaghi, F., Degni, S., \& Innamorati, M. (2011). Sex-specific relationships among attachment security, social values, and sensation seeking in early adolescence: implications for adolescents' externalizing problem behaviour. Journal of Adolescence, 34, 541-554.

Westen, D., Shedler, J., \& Lingiardi, V. (2003). La valutazione della personalità con la SWAP-200 [Personality assessment with the SWAP-200]. Milano: Raffaello Cortina.

Received June 17, 2012

Revision received September 21, 2012

Accepted September 26, 2012 